E3S Web of Conferences 4, 01003 (2014)

DOI: $10.1051 / \mathrm{e} 3$ sconf/20140401003

(C) Owned by the authors, published by EDP Sciences, 2014

\title{
Simulations of the muon flux sensitivity to rock perturbation associated to hydrogeological processes
}

\author{
Fanny Hivert ${ }^{1,2,3, a}$, Ignacio Lázaro ${ }^{1}$, José Busto² ${ }^{2}$ Jean-Pierre Ernenwein ${ }^{2}$, Jürgen Brunner², \\ Jean-Baptiste Decitre ${ }^{1}$, Xavier Martin ${ }^{3}$ and Stéphane Gaffet ${ }^{1,3}$ \\ ${ }^{1}$ LSBB, UMS 3538 Université de Nice, Université d'Avignon, CNRS, Aix-Marseille Université, OCA, \\ La grande combe, 84400 Rustrel, France \\ 2 Aix Marseille Université, CNRS/IN2P3, CPPM UMR 7346, 13288 Marseille, France \\ ${ }^{3}$ Géoazur, Université de Nice Sophia-Antipolis, UMR 7329, CNRS, France
}

\begin{abstract}
Muon tomography is a method to investigate the in-situ rock density. It is based on the absorption of cosmic-ray muons according to the quantity of matter (thickness and density). Numerical simulations are performed in order to estimate the expected muon flux in LSBB Underground Research Laboratory (URL) (Rustrel, France). The aim of the muon measurements in the underground galleries of this laboratory is to characterize the spatial and temporal density variations caused by water transfer in the unsaturated zone of the Fontaine-de-Vaucluse karstic aquifer.
\end{abstract}

\section{Introduction}

Muons are charged particles produced in the atmosphere. Primary cosmic rays, mainly composed of protons $[1,2]$, interact with the atmosphere and produce a huge number of secondary particles, including muons. These muons are particularly interesting because, due to their important mass (i.e., 200 times that of electrons), they are highly penetrating and they are able to propagate down to several hundreds of meters below the surface [3]. Moreover, the attenuation of their flux is proportional to the quantity of matter they cross. Muography is a method to investigate the subsurface, its principle being based on muon absorption to estimate the density of the rock.

This method has been used in several fields since 1955 [4]. Alvarez et al. [5] used it for archeology, to study the internal structure of the Chephren pyramid. It has been particularly developed in volcanology [6-9]. These last years, the projects using muons have diversified: the $\mathrm{CO}_{2}$ storage [10], exploration of Mars [11], etc.

\section{Muon tomography applied to hydrogeology}

The T2DM2 (Temporal Tomography of the Density using the Measurement of Muons) project aims at characterizing the density variations linked to hydrogeological processes. The measurements are

\footnotetext{
${ }^{\text {a }}$ Corresponding author: hivert.fanny@lsbb.eu
}

This is an Open Access article distributed under the terms of the Creative Commons Attribution License 4.0, which permits unrestricted use, distribution, and reproduction in any medium, provided the original work is properly cited. 


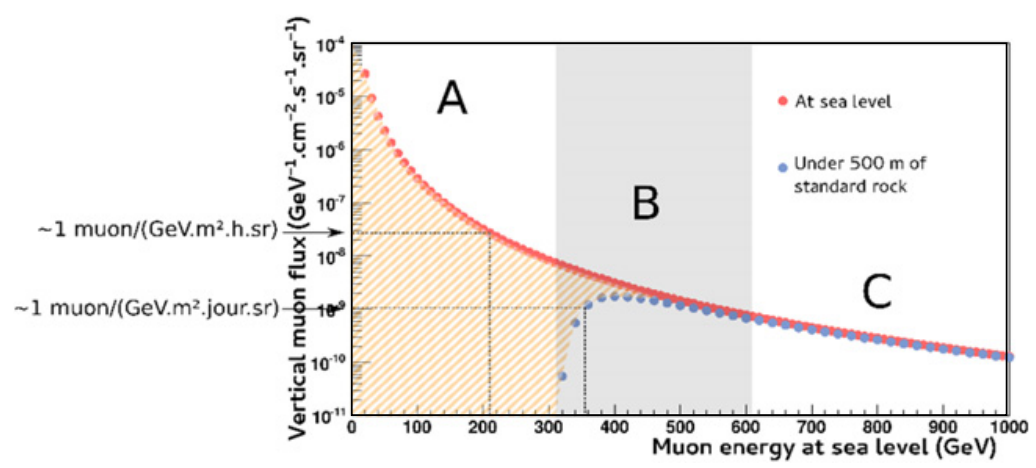

Figure 1. Comparison of the vertical muon fluxes at sea level and at $500 \mathrm{~m}$ depth of standard rock. 3 areas are defined: (A) muons are unable to pass through $500 \mathrm{~m}$ of standard rock; (B) a part of the muons is attenuated by the rock; (C) almost all the muons can reach $500 \mathrm{~m}$ depth of standard rock. The most interesting areas to observe flux variations are $\mathrm{A}$ and $\mathrm{B}$ (hatched area).

carried out in the low noise underground laboratory of Rustrel (LSBB, France). The galleries of this laboratory have a rock overburden up to $500 \mathrm{~m}$ and they are located in the unsaturated zone of the Fontaine-de-Vaucluse karst aquifer. This spring is characterized by an important catchment area that is mostly supplied by rainfall. The $800 \mathrm{~m}$ thick unsaturated area has an important role in the storage and the transfer of the water from the surface to the underlying aquifer. The variable permeability allows a duration of water storage ranging from some days to several months [12]. In such a context, the study of the location and the evolution of the density variations caused by water displacement can provide new information on the operation of this complex karstic system. Moreover the porosity can vary between $0 \%$ and $25 \%$ [13] and consequently the expected density variations may reach $10 \%$.

The carried out simulations, which reflect the LSBB configuration, have a duration fixed to one month, a detection surface to $1 \mathrm{~m}^{2}$ and an angular aperture to $10^{\circ}$.

\section{Simulations of muon flux}

The simulation of muon flux is divided into two parts: it is estimated at sea level and after crossing rock of variable thickness. The surface muon flux is simulated from the Gaisser analytical model [14]. The characteristics of the standard atmosphere constitute the input of the program (notably the opacity of the atmosphere, the altitude of muon production, etc.). The surface muon flux strongly decreases when the particle energy grows (Fig. 1). The survival probabilities of muons inside the rock are calculated with the MUSIC code [15], an open access Monte Carlo code dedicated to the simulation of muon propagation through rock or water. Muon survival probabilities are calculated according to the matter characteristics (thickness, density, atomic composition and radiation length) and the particle energy. They reduce with rising amount of crossed matter as well as with decreasing initial energy. Thus, is needed a higher critical energy to pass through the rock. Consequently, the integral muon flux decreases as well. The flux can be reduced by several orders of magnitude (Fig. 1) and that's why the most important limitation in muon tomography is the accumulated muon statistics. The flux variations that can be distinguished with reasonable statistic significance have to be studied.

\section{Sensitivity to density variations of rocks}

In order to estimate the sensitivity of muography, various rock densities are tested. They are compared to the standard rock defined in MUSIC with a density of $2.65 \mathrm{~g} . \mathrm{cm}^{-3}$ and an atomic composition $\mathrm{Z}=11$ 
a

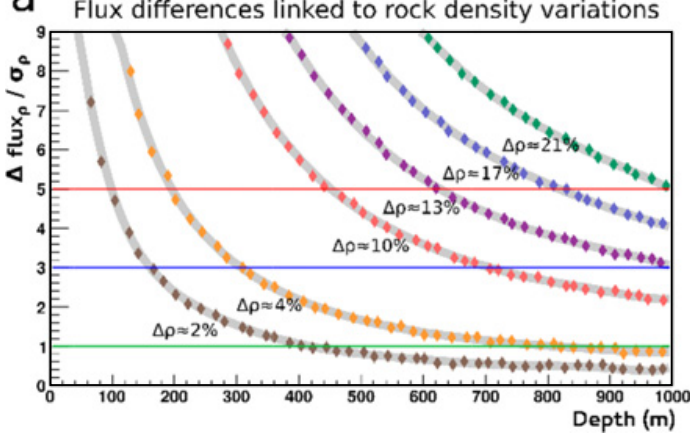

b Flux differences linked to rock composition variations

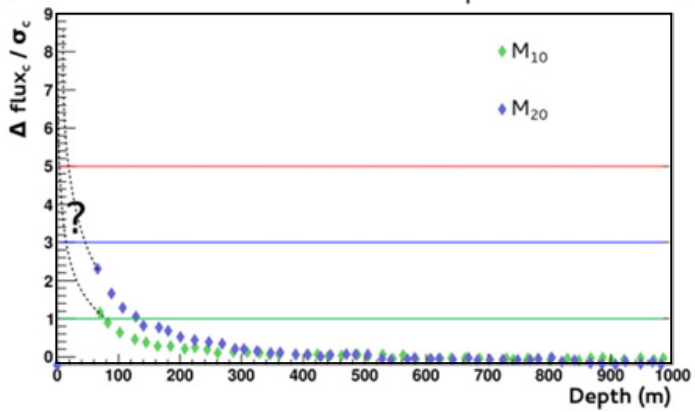

Figure 2. Influences of density (a) and composition (b) variations on the muon flux. The red, blue and green straight lines are respectively the values $1 \sigma, 3 \sigma$, and $5 \sigma$ of significance. (a) The flux differences caused by density variations (in percentages) are fitted by the shaded curves. (b) The muon flux variations are studied for 3 rock compositions $\left(0 \%, 10 \%\left(\mathrm{M}_{10}\right)\right.$ and $20 \%\left(\mathrm{M}_{20}\right)$ of $\left.\mathrm{H}_{2} \mathrm{O}\right)$ with a constant density $\left(2.40 \mathrm{~g} . \mathrm{cm}^{-3}\right)$. These muon fluxes are calculated for a duration of one month, a detection surface of $1 \mathrm{~m}^{2}$ and an angular aperture of $10^{\circ}$. The results for depths lower than $60 \mathrm{~m}$ are extrapolated from deeper simulations (dashed lines).

and $\mathrm{A}=22$. The flux variations are computed for several density variations between this standard rock and rocks with other densities. The following equation 1 represents the variations of the flux $\left(\Delta\left(\Phi_{\rho}\right)\right)$ divided by the errors of this flux $\left(\sigma_{\rho}\right)$.

$$
\Delta\left(\phi_{p}\right) / \sigma_{p}=\left|\phi_{\text {standard }}-\phi_{\Delta p}\right| / \sqrt{\sigma_{\text {standard }}^{2}+\sigma_{\Delta p}^{2}} .
$$

Where $\Phi_{\text {standard }}$ and $\Phi_{\Delta \rho}$ are the muon fluxes in $\mathrm{GeV}^{-1} \cdot \mathrm{cm}^{-2} \cdot \mathrm{s}^{-1} \cdot \mathrm{sr}^{-1}$ for the standard rock and the rocks with other densities respectively, $\sigma_{\text {standard }}$ and $\sigma_{\Delta \rho}$ are their respective errors. The density differences are presented in percentages and for depths ranging from $60 \mathrm{~m}$ to $1000 \mathrm{~m}$ (Fig. 2a). The minimum depth is fixed to $60 \mathrm{~m}$ because lower depths require a more specific study that is not in the context of this paper. The simulations are performed until $1000 \mathrm{~m}$ to take into account the non-vertical incidence muons at the deepest part of the LSBB $(\sim 500 \mathrm{~m})$. The results are compared to $1 \sigma, 3 \sigma$ and $5 \sigma$ of significance. The maximum depth limitation range is defined when the significance of the flux variations is between $3 \sigma$ and $5 \sigma$. For example, density variations of about $2 \%$ can be detected with $3 \sigma$ significance until $\sim 160 \mathrm{~m}$. The same significance is reachable until $\sim 320 \mathrm{~m}$ for $4 \%$ and $\sim 720 \mathrm{~m}$ for $10 \%$. Density variations higher than $15 \%$ are observable until the maximum tested depth: $1000 \mathrm{~m}$.

\section{Sensitivity to slight variations of the atomic composition}

The nature of rock atoms may also have an influence on the muon flux. The atomic composition variations are applied by changing the $Z$ (atomic number) and $A$ (mass number) mean values of rock elements in MUSIC code. Three rock compositions have been tested: a dry rock and rocks with $10 \%$ $\left(M_{10}\right)$ and $20 \%\left(M_{20}\right)$ of $\mathrm{H}_{2} \mathrm{O}$. For these simulations, the density is kept constant at $2.40 \mathrm{~g} . \mathrm{cm}^{-3}$. The simulations focus on composition variations only associated to water saturation conditions. In the same way as for the density tests, the flux differences $\left(\Delta\left(\Phi_{C}\right)\right)$ between the dry rock and the rocks with $10 \%$ and $20 \%$ of $\mathrm{H}_{2} \mathrm{O}$ are divided by their errors $\left(\sigma_{C}\right)$ and compared to $1 \sigma, 3 \sigma$ and $5 \sigma$ (Eq. (2)).

$$
\Delta\left(\phi_{c}\right) / \sigma_{c}=\left|\phi_{d r y}-\phi_{10 / 20}\right| / \sqrt{\sigma_{d r y}^{2}+\sigma_{10 / 20}^{2}} .
$$


Where $\Phi_{d r y}$ and $\Phi_{10 / 20}$ are the muon fluxes in $\mathrm{GeV}^{-1} \cdot \mathrm{cm}^{-2} \cdot \mathrm{s}^{-1} \cdot \mathrm{sr}^{-1}$ for the dry rock and the rocks with $10 \%$ and $20 \%$ of $\mathrm{H}_{2} \mathrm{O}, \sigma_{d r y}$ and $\sigma_{10 / 20}$ are their respective errors. No flux differences higher than $3 \sigma$ are obtained for the tested rocks, at depths ranging from $60 \mathrm{~m}$ to $1000 \mathrm{~m}$ (Fig. 2b). Compared to the density effect, the composition influence on muon flux is negligible. This conclusion is suitable only for classical rocks, the presence of high- $\mathrm{Z}$ materials leading to important variations of the muon flux.

\section{Discussion and prospects}

The hydrogeological processes that take place in karstic aquifer are complex and not well known. The LSBB URL provides a perfect location in the unsaturated area of Fontaine-de-Vaucluse to study these processes. Numerical simulations prove that muography is suitable to image the spatial and temporal density variations caused by water transfer. The insensitivity to rock composition variations, compared to the density, demonstrates that muography is a direct measurement of the in-situ rock density.

Currently, a particular attention is paid on the scattering of the muons inside the rock. The idea is to estimate the scattering processes and their impact on the muon flux in order to avoid mistakes on the measurement interpretations. These processes must be studied especially because Micromegas - TPC (Time Projection Chambers) telescopes will be used for the T2DM2 project [16]. These detectors have the spatial and angular high resolutions needed to image the thin geological structures.

Since December 2013, the first muon flux measurements are performed in the LSBB underground galleries with liquid scintillator tanks. This detection system will then be used to calibrate the Micromegas telescopes.

Muography is here applied to hydrogeology but it has already been used in several fields and a lot of others are expected in the next years. Tomography based on cosmic-ray muons is a complementary technique to the standard geophysical methods (e.g., seismic imaging, electric prospection or gravimetry) and can be used for coupled inversions.

The authors want to acknowledge the LSBB underground laboratory team and the Antares group of the CPPM. RD51 collaboration of the CERN and the CEA-IRFU for their work on Micromegas detectors. This work is supported by the Fondation MAIF, CNRS-INSU, Nice University and FEDER. Authors thank anonymous reviewers for their helpful comments.

\section{References}

[1] Fernow, R.C. Introduction to experimental particle physics. Cambridge Univ. Press, 421pp (1986).

[2] Belloti, R. et al. Phys. Rev., D60, 052002 (1999).

[3] Boezio, M. et al. Astrophys. J., 518, 457-472 (1999).

[4] George, E. P. Commonwealth Engineer, 455-457 (1955).

[5] Alvarez, L.W. et al. Science, 167, 832-\& (1970).

[6] Nagamine, K. et al. NIM in Physics Research Section A, 604, 738-746 (1995).

[7] Tanaka, H.K.M. et al. Earth and Planetary Science Letters, 263, 104-113 (2007).

[8] Lesparre, N. et al. Geophys. J. Int., 183, 1348-1361 (2010).

[9] Carloganu, C. et al. Geoscientific Instrumentation Methods and Data Systems, 2, 55-60 (2013).

[10] Kudryavtsev, V. et al. International journal of greenhouse gas control, 11, 21-24 (2012).

[11] Kedar, S. et al. Geoscientific Instrumentation Methods and Data Systems, 2, 157-164 (2013).

[12] Garry, B. Thèse de doctorat, Univ. d'Avignon et des Pays du Vaucluse, Avignon, 218pp (2007).

[13] Fournier, F. et al. Geophysics, 76, E211-E226 (2013).

[14] Gaisser, T. Cosmic rays and particle physics. Cambridge University Press, Cambridge (1990).

[15] Kudryavtsev, V. Computer Physics Communications, 180, 339-346 (2009).

[16] Lázaro, I. et al. proceedings of i-DUST conference, Rustrel, France (2014) (in press). 